\title{
IDENTIFYING MISSED OPPORTUNITIES FOR EARLY INTERVENTION AMONG HIV- INFECTED PAEDIATRIC ADMISSIONS AT CHRIS HANI BARAGWANATH HOSPITAL, SOWETO, SOUTH AFRICA
}

\author{
Angela Dramowski ${ }^{1}, M B C h B$, FCPaed, MMed (Paed), Cert Paed ID, DCH \\ Ashraf Coovadia ${ }^{2}, M B C h B$, FCPaed, Dip HIV Man, DCH \\ Tammy Meyers ${ }^{3}, M B C h B$, FCPaed, MMed (Paed), DTM\&H \\ Ameena Goga ${ }^{4}, M B C h B, F C P a e d, D T M \& H, M S c(M C H), M S c$ (Epid) \\ ${ }^{I}$ Department of Paediatrics and Child Health, Tygerberg Children's Hospital and Stellenbosch University, Tygerberg, W Cape \\ ${ }^{2}$ Department of Paediatrics and Child Health, Rahima Moosa Mother and Child Hospital, Faculty of Health Sciences, \\ University of the Witwatersrand, Johannesburg \\ ${ }^{3}$ Department of Paediatrics and Child Health, Chris Hani Baragwanath Hospital, Faculty of Health Sciences, University of the Witwatersrand \\ ${ }^{4}$ South African Medical Research Council, Pretoria
}

Background and design. HIV is a major contributor to childhood morbidity and mortality in South Africa. We describe HIV prevalence, disease profile, outcome and missed opportunities for early intervention in a cohort of HIV-infected children admitted to Chris Hani Baragwanath Hospital's general paediatric wards between 1 October 2007 and 31 December 2007.

Results. Of 1510 admissions, 446 (29.5\%) were HIV infected. Many children (238, 54.1\%) were newly diagnosed in hospital and most had advanced HIV disease (405, 92\%). The principal admission diagnoses were pneumonia $(165,37.5 \%)$, gastro-enteritis $(97,22 \%)$, sepsis $(86,19.5 \%)$ and tuberculosis $(92,21 \%)$. Of children identified as HIV infected before admission, 128/202 (63.4\%) were not accessing antiretroviral treatment (ART), although $121 / 128(94.5 \%)$ met ART eligibility criteria. Of 364 ART-naïve eligible children, only $15(4.1 \%)$ were commenced on ART as inpatients. Problems with PMTCT implementation in infants under 6 months $(N=166)$ included lack of maternal antenatal HIV testing $(51,30.7 \%)$; poor uptake of maternal/infant nevirapine prophylaxis $(60,36.2 \%)$; limited use of co-trimoxazole (CTX) prophylaxis (44/147, 29.9\%); and delayed infant HIV polymerase chain reaction testing $(98 / 147,87.5 \%)$. Of infants known to be HIV infected prior to hospitalisation, $37 / 51(73 \%)$ had not initiated ART. The in-hospital case fatality rate (CFR) among HIV-infected children was triple that of the combined HIV-uninfected, exposed and unknown group (12\% v. 3.6\%). Infants $<12$ months of age accounted for $73.6 \%$ of all HIV-related deaths (CFR 17.1\%).

Conclusions. HIV remains highly prevalent and contributes to significant in-hospital mortality. Missed opportunities for PMTCT, HIV diagnosis and ART initiation are frequent. Interventions to optimise paediatric HIV outcomes should target maternal HIV diagnosis, early infant diagnosis, uptake of CTX prophylaxis and prompt initiation of ART, especially among infants. Hospitalised ART-eligible children should be prioritised for inpatient initiation of ART.

South Africa has 5.6 million people living with HIV, including approximately 280000 children $^{1}$ who suffer disproportionate morbidity and double the mortality of their HIV-uninfected counterparts. ${ }^{2,3}$ Past progress in reducing national child mortality has been reversed by paediatric HIV, with under-5 mortality rates (U5MR) increasing from 56 to 67 deaths per 1000 live births between 1990 and 2008. ${ }^{4}$ Much of the increase in the U5MR can be accounted for by deaths in young HIVinfected infants, many of whom progress rapidly to AIDS and death from opportunistic infections, without early initiation of antiretroviral therapy (ART). ${ }^{5,6}$

South Africa introduced the prevention of mother-tochild transmission (PMTCT) and ART programmes nationwide in 2001 and 2004, respectively. The national PMTCT guidelines at the time of this study (2007) recommended HAART for pregnant women with a CD4 count $<200$ cells/ $\mu$ l or zidovudine (AZT) from 28 weeks' gestation plus intrapartum single-dose nevirapine 
(sdNVP) at CD4 counts $>200$ cells $/ \mu$ l. All infants were scheduled to receive sdNVP at delivery. The policy for early infant diagnosis (EID) at the time recommended HIV polymerase chain reaction (PCR) testing at 6 weeks of life, and for breastfed babies a repeat HIV PCR test 6 weeks after complete cessation of breastfeeding. ART initiation was recommended for any child with clinically advanced disease (WHO HIV stage 3 or 4 ) or immunological compromise (CD $4<20 \%$ in children less than 18 months of age and CD4 $<15 \%$ in children over 18 months). ${ }^{7}$ Despite national implementation of these programmes, coverage and uptake of PMTCT, EID and paediatric ART programmes were highly variable between provinces. ${ }^{8}$ More recently, improved coverage of these interventions has been achieved; however, many infants miss entry points for the PMTCT programme and routine HIV testing. Others may be identified but are lost from the system or become ill before ART is initiated. ${ }^{9}$ These children typically present to hospital with advanced HIV disease and consequently have high mortality rates. ${ }^{10,11}$ Chris Hani Baragwanath hospital $(\mathrm{CHBH})$ is South Africa's largest public sector hospital, delivering care to the burgeoning urban and low-income population of Soweto, Johannesburg. Paediatric HIV prevalence at $\mathrm{CHBH}$ (described in two previous studies prior to the implementation of PMTCT programmes) rose from 3\% to $19 \%{ }^{12}$ to $29 \%{ }^{13}$ between 1992 and 1996 . Over the same period the proportion of paediatric in-hospital mortality accounted for by HIV increased dramatically from $6.7 \%$ to $46.1 \% .{ }^{12}$ Similar trends in HIV prevalence and HIV-related mortality in other South African hospitals have been reported from a national data collection programme, Child PIP. ${ }^{10,11}$ We report on paediatric HIV period prevalence, disease profile and outcome of children admitted to $\mathrm{CHBH}$ in the last quarter of 2007, several years after introduction of national PMTCT and paediatric HIV management programmes. In addition we describe missed opportunities for HIV prevention, diagnosis and medical intervention among this cohort of HIV-infected children.

\section{METHODS}

Ethics. The study was approved by University of the Witwatersrand Human Research Ethics Committee (reference No. M080202).

Study site. The study was undertaken at $\mathrm{CHBH}$, Soweto, Johannesburg, in the Gauteng province of South Africa. This 2 964-bed referral hospital is the only public hospital serving approximately 3.5 million Sowetans and accepts referrals from local primary health care clinics, regional hospitals in Gauteng and neighbouring provinces of South Africa. In 2010, the estimated HIV prevalence in Gauteng province was $10.5 \%{ }^{14}$ The province's health sector, although challenged by high TB and HIV prevalence, is relatively well resourced and staffed when compared with other areas of South Africa. The hospital and all referring institutions follow national PMTCT and HIV management guidelines. A dedicated paediatric HIV clinic on the hospital premises (Harriet Shezi clinic) provides outpatient services to more than $3500 \mathrm{HIV}$-infected children and the Perinatal HIV Research Unit (PHRU) provides PMTCT support in the Soweto area.
Study design and sampling. A cross-sectional retrospective review of all children (from birth to 14 years of age) hospitalised in the general paediatric wards between 1 October 2007 and 31 December 2007 was performed. Newborns with reactive HIV PCR test results during their stay in the neonatal unit were not included in this study, unless they were subsequently admitted to the general paediatric wards during the 3-month study period. Patient admission numbers and profile over this 3-month period did not differ significantly from the preceding three-quarters of 2007. Two populations were of interest (Fig. 1). Firstly, admission register lists of all hospitalised children were used to determine HIV status (collected from laboratory and/ or hospital records) and calculate HIV period prevalence. Secondly, individual patient records for children identified as HIV infected were reviewed. Monthly paediatric mortality reports were used to calculate in-hospital mortality. Selfreported data on PMTCT coverage were analysed only for HIV-infected infants less than 6 months of age, to minimise information recall bias. For analysis of missed opportunities in provision of co-trimoxazole (CTX) prophylaxis and uptake of EID, infants $<6$ weeks were excluded.

Determination of HIV status. All HIV tests were performed by the accredited National Health Laboratory Service (NHLS). A reactive HIV-DNA PCR test confirmed HIVinfected status in children under 18 months. A reactive HIV enzyme-linked immunosorbent assay (ELISA) confirmed HIV-infected status in children older than 18 months of age. Mothers' self-reported HIV status (as documented in hospital records) was used. Four definitions were used: HIV uninfected refers to the infant or child being confirmed HIV uninfected; HIV infected refers to the infant or child being confirmed HIV infected; if the mother's status was reportedly HIV infected and her infant had no HIV PCR result, the infant's status was classified as HIV exposed. If maternal HIV status was unknown or uninfected and the child had no HIV test result in laboratory or folder records, the status was classified as HIV unknown.

Case definitions and reference classifications. In cases where laboratory confirmation was not obtained, a working diagnosis was based on clinical suspicion and the World Health Organization (WHO) published case definitions ${ }^{15}$ for the following conditions: Pneumocystis jiroveci pneumonia (PCP), ${ }^{16,17}$ pulmonary tuberculosis (pTB), cytomegalovirus (CMV) pneumonitis or disseminated disease, septicaemia, meningitis and urinary tract infection. HIV disease severity was assessed using the WHO clinical staging system for children ${ }^{15}$ and nutritional status using the WHO 2006 growth standards ${ }^{18}$ for calculation of z-scores in children under 60 months of age. Calculation of ART eligibility was based on the South African national guidelines $(2005)^{7}$ at the time of the study, using immunological (CD4\% $<20 \%$ under 18 months of age; CD4\% <15\% above 18 months of age) and clinical criteria (WHO stage 3 and 4 disease) only. The WHO and the South African Department of Health published extensively revised guidelines for child ART initiation in 2010.

Statistical analysis. Data were analysed in SAS version 9.1 (SAS Institute Inc., Cary, NC, USA). Crude HIV period prevalence was calculated from ward records 
as total HIV-infected admissions/total admissions. Case fatality rates in the HIV-infected and combined HIVuninfected, HIV-unknown and HIV-exposed groups were calculated from total number of deaths/total number of admissions for each group. For the PMTCT sub-analysis, frequency calculations were performed for maternal HIV status, sdNVP exposure, CTX prophylaxis and place of birth. The uptake of PMTCT interventions was then compared by maternal HIV status grouping using the chi-square test. A $p$-value of $<0.05$ was considered to be statistically significant.

\section{RESULTS HIV PREVALENCE}

Of 1510 children admitted during the 3-month study period, 446 (29.5\%) were HIV infected, 780 (51.7\%) were HIV uninfected, 57 (3.8\%) were HIV exposed and $227(15 \%)$ were of unknown HIV status (Fig. 1). For the 446 children identified as HIV infected, 440 (98.7\%) individual patient records were located.

\section{HIV-INFECTED CHILDREN: PROFILE OF STUDY POPULATION}

Table I outlines the demographic and disease profile of the 440 HIV-infected children. Almost 93\% had advanced HIV disease (WHO stage 3 or 4 ) and $55.3 \%$ of children $<5$ years of age had severe malnutrition. Across all age groups, $225 / 320$ children $(70.3 \%$ ) had severe immune suppression.

\section{REASON FOR HOSPITALISATION}

Infectious disease was the principal reason for hospitalisation (Table I). Lower respiratory tract infections (including presumed PCP and presumed Cytomegalovirus pneumonitis) accounted for the majority of admissions, with the highest prevalence among infants. One hundred and ten children (25\%) had a confirmed bacterial, viral or fungal infection during their hospital admission. Streptococcus pneumoniae and CMV were the most common bacterial and viral pathogens isolated.

\section{MISSED OPPORTUNITIES}

\section{Prevention of mother-to-child transmission of HIV}

Self-reported maternal HIV status and sdNVP exposure were analysed in HIV-infected infants under the age of 6 months $(n=166)$. Uptake of CTX prophylaxis and EID were analysed only for infants $>6$ weeks of age $(n=147)$ so as to be consistent with programme guidelines. Fig. 2 highlights the multiple levels of missed opportunities for PMTCT implementation in infants ( $<6$ months of age) whose mothers reported their status as HIV infected versus HIV uninfected versus HIV unknown. Lack of maternal antenatal HIV testing was documented among 51/166 $(30.7 \%)$ mothers. There was poor uptake of maternal/ infant NVP prophylaxis $(60 / 166,36.2 \%)$. Usage of CTX prophylaxis was limited (44/147, 29.9\%) and in most cases infant HIV PCR testing was delayed or lacking (98/147, $87.5 \%$ ). There was no association between place of birth ( $\mathrm{CHBH}$ versus clinic/other hospital/home) and access to NVP or CTX ( $p=0.1288$ and $p=0.5818$, respectively). Of the 147 HIV-infected infants 6 weeks $-<6$ months of age, $51(34.7 \%)$ were known to be HIV infected while the remainder, 96 (65.3\%), were newly diagnosed (i.e. had not previously had HIV PCR testing) at the time of hospital admission. Sixty-seven (45.6\%) of the 147 infants received no PMTCT interventions at all. Only 20/147 (13.6\%) infants received all recommended interventions, i.e. NVP and CTX and EID. Table I reflects additional missed opportunities for the provision of CTX prophylaxis among other categories of the study population.

HIV diagnosis and antiretroviral therapy (ART) eligibility and uptake Most children, 238/440 (54.1\%), were newly diagnosed at the time of hospitalisation at $\mathrm{CHBH}$. Newly diagnosed children were younger than those already known to be HIV infected (median 6 v. 12 months of age) $(p=0.001)$. Children known to be infected and already on ART had a median treatment duration of 2 months and were significantly older than ART-naïve children (median age 38 v. 7 months) $(p<0.0001)$. Of children known to be infected but not on ART $(128 / 202,63.4 \%)$, nearly all $(121 / 128,94.5 \%)$ were eligible for ART based on advanced disease stage. Of 364 ART-naïve eligible children, only $15(4.1 \%)$ were commenced on ART as inpatients (Fig. 1). Table II compares eligibility for ART with actual ART uptake among children who died.

\section{OUTCOME OF HOSPITALISATION}

Median duration of hospital stay was 7 days (interquartile range (IQR) 4 - 10.) Fifty-three children died (Table I) with a median duration of stay before death of 4 days (IQR 2 - 7.8). Only 13 children (3.0\%) were admitted to an ICU or underwent mechanical ventilation in the highcare area of the acute admissions ward. Ten of these 13 children (76.9\%) survived to hospital discharge.

Fifty-eight per cent of all paediatric deaths (53/91) occurred among the HIV-infected group. The overall case fatality rate in the HIV-infected children was 53/440 (12.0\% (95\% confidence interval (CI) 9.2 - 15.5\%)). In contrast, the case fatality rate in the HIV-uninfected, HIV-exposed and HIV-unknown group over the study period was $38 / 1064(3.6 \%(95 \% \mathrm{Cl} 2.5-4.9 \%))$. The highest case fatality rate by age group was in infants aged less than 12 months (Table I). The most prevalent causes of death included pneumonia/suspected PCP $(18 / 53,34 \%)$, TB $(14 / 53,26.3 \%)$ and gastro-enteritis $(5 / 53,9.4 \%)$. The inpatient mortality rate did not differ significantly between children receiving ART at the time of hospitalisation $(7.9 \%, 6 / 76)$ versus ART-naïve children $(13.6 \%, 47 / 345)(p=0.17$, odds ratio 0.54$)$.

\section{DISCUSSION HIV PREVALENCE}

This paper reports the first published data on paediatric HIV prevalence, disease profile and outcomeat $\mathrm{CHBH}$ subsequent to widespread implementation of PMTCT and paediatric HIV management programmes. The HIV period prevalence of $29.5 \%$ was almost identical to that found in 1996. However, the true HIV prevalence remains unquantified, since both cohorts had large numbers of untested children. Several years after national roll-out of PMTCT and ART programmes, there is therefore little evidence of a decreasing impact of paediatric HIV at $\mathrm{CHBH}$. Possible explanations for this could include increasing antenatal HIV prevalence in Gauteng province (15.5 - 29.8\% between 1996 and 2009$)^{19}$ with more vertical infections; failure of sdNVP PMTCT regimens; the 


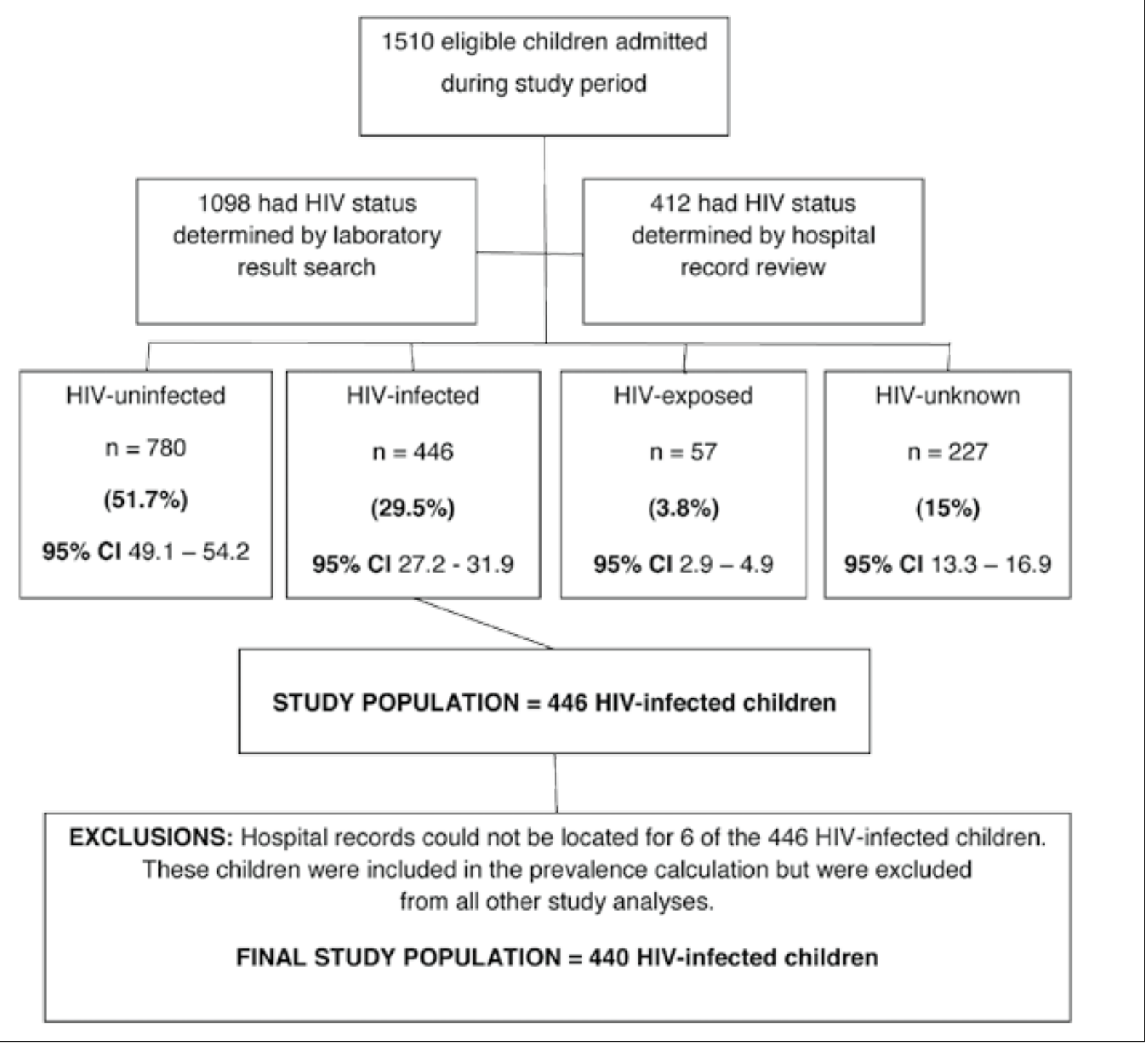

Fig. 1. Determination of HIV prevalence and study population and ART uptake among 440 HIV-infected children.

possibility of an even higher (undocumented) peak paediatric HIV prevalence reached between 1996 and 2007; poor PMTCT coverage; improved survival rates (particularly in older HIV-infected children on ART); and the establishment of a dedicated paediatric HIV clinic at $\mathrm{CHBH}$ (pooling children with complicated HIV disease).

\section{MISSED OPPORTUNITIES}

Prevention of mother-to-child transmission of HIV

A major limitation of the chosen study design is possible maternal recall bias regarding PMTCT interventions. In addition, reported maternal HIV status could not be verified in all cases. Healthcare workers' documentation of PMTCT interventions was poor. In order to decrease missed opportunities among HIV-exposed infants, all PMTCT interventions should be clearly explained to caregivers and documented in all patient records, especially the infants' Road to Health Card (RTHC). This is the most important linkage and communication tool for paediatric healthcare providers, especially when children access care at multiple facilities.

Uptake of PMTCT interventions was poor, with almost half of infants under 6 months of age receiving no PMTCT interventions at all. Of particular concern was the large proportion of mothers who reported their status as HIV uninfected $(15.1 \%)$ or HIV unknown/untested $(30.7 \%)$. There are several possible explanations for the $15.1 \%$ of mothers who reported their status as HIV uninfected: a negative test in early pregnancy with subsequent seroconversion before delivery (3\% reported seroconversion of pregnant women); ${ }^{20}$ postnatal HIV infection with breastfeeding transmission; or fear of stigmatisation after disclosure of HIV status. Antenatal identification of HIV-infected pregnant women is the gateway to a successful PMTCT programme, since all other interventions rely on this key step. Optout antenatal HIV testing, and repeat HIV testing late in pregnancy, during labour and at immunisation services, would maximise identification of HIV-infected mothers. In addition, clear recording of maternal HIV status on the infant's RTHC would increase awareness of HIV exposure and hopefully prompt prescription of CTX and uptake of infant HIV PCR testing. This would allow for earlier infant diagnosis and rapid ART initiation, resulting in improved infant outcomes.

The poor uptake rates reported for sdNVP and CTX prophylaxis in this study are alarming. Possible factors contributing to this problem include mothers who test 


\section{TABLE I. DISEASE PROFILE OF HIV-INFECTED CHILDREN ( $N=440)$}

\section{Gender}

Male

Female

$234(53.2 \%)$

Age category

$\leq 11$ months

12 - 35 months

$206(46.8 \%)$

36 - 59 months

$228(51.8 \%)$

$85(19.3 \%)$

$\geq 5$ years

$29(6.6 \%)$

$98(22.3 \%)$

Timing of HIV diagnosis

Newly diagnosed

Known HIV-infected

$238(54.1 \%)$

$202(45.9 \%)$

WHO HIV stage

I

II

III

IV

$$
3(0.7 \%)
$$

$29(6.6 \%)$

$188(42.7 \%)$

$220(50 \%)$

Median (IQR) weight-for-age z-score (WAZ) ${ }^{*}(n=342)$

$\leq 11$ months $(n=228)$

12 - 35 months $(n=85)$

36 - 59 months $(n=29)$

No. 0 - 59 months with WAZ $-3--2(\%)$

No. 0 - 59 months with WAZ <-3 (\%)

Median (IQR) CD4 percentage by age group ${ }^{\dagger}(n=320)$

$\leq 11$ months $(n=155)$

12 - 35 months $(n=57)$

36 - 59 months $(n=23)$

$\geq 5$ years $(n=85)$

$-3.53(-4.59--2.44)$

$-2.69(-3.60--1.43)$

$-1.89(-3.03--1.41)$

$56(16.4)$

$189(55.3)$

Median (IQR) CD4 absolute count (cells/ $\mu$ l) by age $\operatorname{group}^{\dagger}(N=320)$

$\leq 11$ months $(n=155)$

12 - 35 months $(n=57)$

36 - 59 months $(n=23)$

$\geq 5$ years $(n=85)$

Severe immunosuppression (all ages) ${ }^{\ddagger}$

$18.6(12.8-28)$

$16.2(9.6-23)$

$9.1(6.3-15.3)$

$8.0(4.5-14.7)$

$700(361-1294)$

$565(422$ - 909)

$414(197-863)$

$171(45-379)$

$225 / 320,70.3 . \%$

No. (\%) of children receiving CTX prophylaxis

All HIV-infected infants $<12$ months of age

$73 / 228(32 \%)$

$86 / 127(67.7 \%)$

$53 / 55(96.4 \%)$

Known to be HIV-infected and on ART $<12$ months

$165(37.5 \%)$

$97(22 \%)$

$92(21 \%)$

$86(19.5 \%)$

(including septicaemia $(n=48)$, meningitis $(n=23)$, and urinary tract infection $(n=15)$

Outcome of hospitalisation

Discharged alive

Died

$319(72.5 \%)$

Transferred to a step-down hospital facility

$53(12 \%)$

$68(15.5 \%)$

Deaths by age group (case fatality rate $\$, 95 \% \mathrm{Cl}$ ) by age group $(n=53)$

$\leq 11$ months

$12-35$ months

$39(17.1 \%, 12.5-22.6)$

36 - 59 months

$4(4.7 \%, 1.3-11.6)$

$2(6.9 \%, 0.8-22.8)$

$\geq 5$ years

$8(8.2 \%, 3.6-15.5)$

* Calculation of WAZ scores was only done for children 0 - 59 months of age $(n=342)$.

${ }^{\dagger}$ CD4-positive T-cell counts and percentages were analysed in a subgroup of the study population who had had CD4 testing at any point 1 month before, during or after hospitalisation $(n=320 / 440)$.

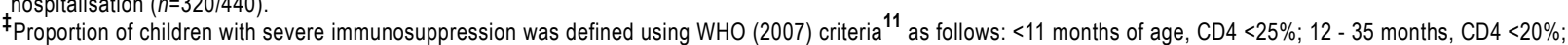
36 - 59 months, CD $4<15 \%$; $>5$ years, CD $4<200$ or $<15 \%$.

$\S$ Case fatality rate was calculated as total number of deaths per age group/total number of admissions per age group.

at a clinic and then deliver in hospital; 'cryptic' written communication between health facilities about patients' HIV status in an attempt to maintain confidentiality; women's reluctance to disclose HIV status due to stigma; and health care workers' reluctance to offer HIV testing or to enquire about HIV status. Failure to provide CTX prophylaxis represents a major missed opportunity to prevent early mortality from PCP, as demonstrated in this cohort with $15 \%$ of deaths ascribed to PCP. Since 2007 there has been considerable improvement (but 


$\begin{array}{llll}\text { Age categories } & \begin{array}{l}\text { No. (\%) with immunological } \\ \text { criteria qualifying for ART* }\end{array} & \begin{array}{l}\text { disease (stage 3 or 4) qualifying } \\ \text { for ART }\end{array} & \begin{array}{l}\text { actually receiving ART at } \\ \text { time of death }\end{array} \\ \begin{array}{lll}\text { All deaths } \\ (n=53)\end{array} & 50 / 53 & 6 / 50 \\ \left(6 / 27^{\dagger}\right. & (94.3) & (12) \\ \text { Deaths }<18 \text { months } & 10 / 17^{\dagger} & 38 / 40 & 6 / 38 \\ (n=40) & (58.8) & (95) & (15.8) \\ \text { Deaths }>18 \text { months } & 8 / 10^{\dagger} & 12 / 13 & 0 / 12 \\ (n=13) & (80) & (92.3) & (0) \\ \text { *CD4 percentage <20\% for children <18 months old, CD4 <15\% for children >18 months old. } & \\ { }^{*} \text { The denominators differ from the overall group denominators because only a proportion of the } 53 \text { HIV-infected children who died had recent CD4 percentage results } \\ \text { available. }\end{array}$

No. (\%) with clinically advanced disease (stage 3 or 4 ) qualifying for ART

$50 / 53$

$(94.3)$

(95)

$12 / 13$
No. (\%) of eligible children actually receiving ART at time of death

$6 / 50$

$0 / 12$

(0)

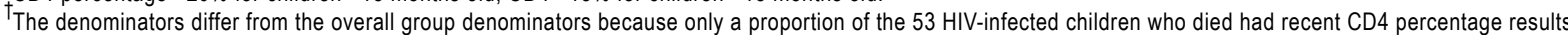
available.

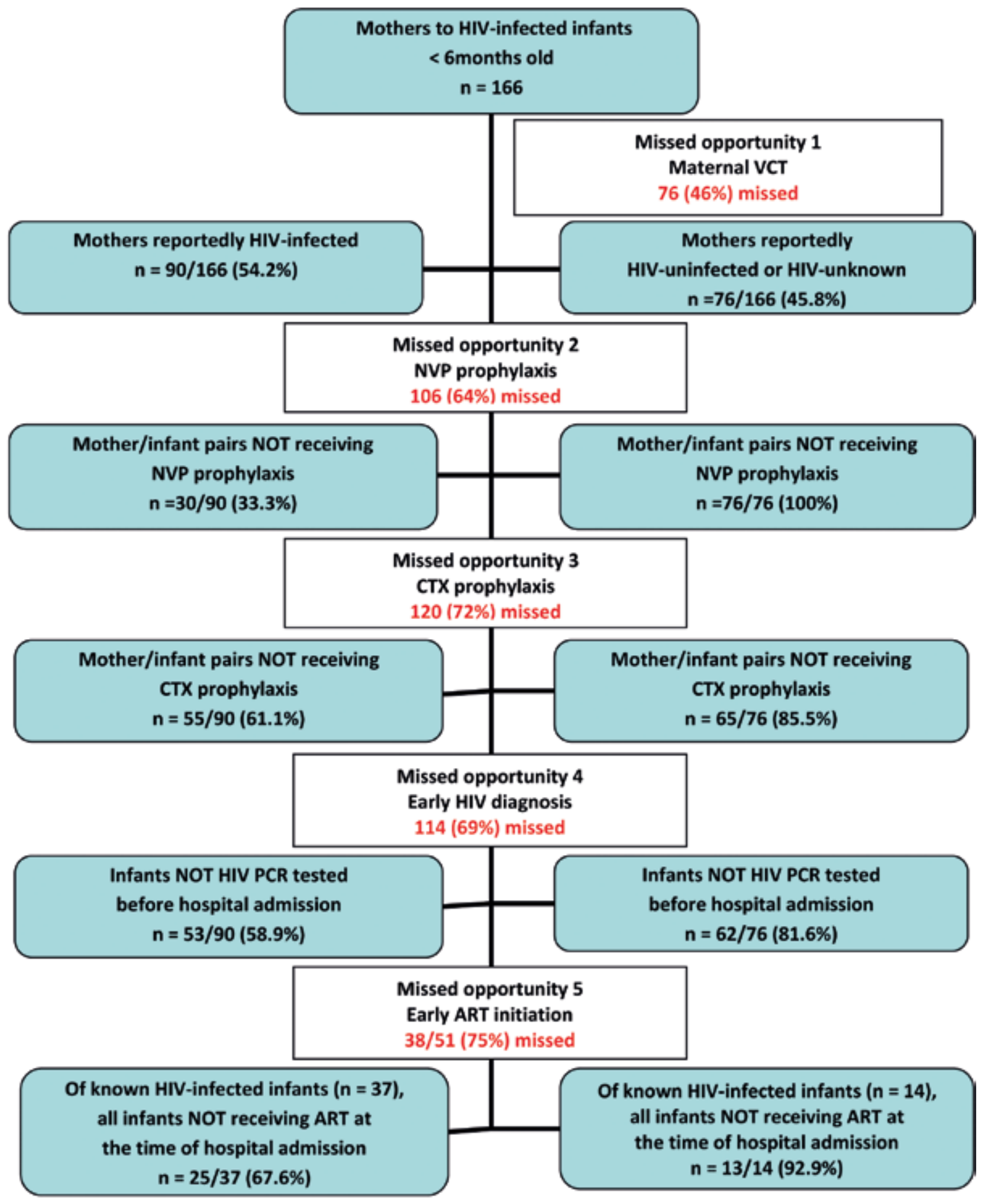

Fig. 2. Missed opportunities for PMTCT implementation, CTX prophylaxis, EID and ART initiation in infants (<6 months). 
increased complexity) in PMTCT regimens. However, these changes will be not be effective unless universal uptake of PMTCT, CTX prophylaxis and early infant diagnosis is achieved. High loss to follow-up of HIVexposed infants remains a major problem in the PMTCT programme, both at $\mathrm{CHBH}$ and at a national level as reported by previous studies. ${ }^{21,22}$

\section{HIV diagnosis and antiretroviral therapy eligibility and uptake} Despite calls for universal testing, $15 \%$ of children admitted to $\mathrm{CHBH}$ had no documentation of HIV exposure status or HIV testing. In a subgroup (3.8\%) noted to be HIV exposed and symptomatic (requiring hospitalisation), HIV PCR testing was not performed. Although usually recommended at 4 - 6 weeks of age, immediate HIV PCR testing should be performed in symptomatic HIV-exposed infants regardless of age so as to expedite ART initiation. ${ }^{23}$ Maternal HIV status, PMTCT interventions and results of HIV PCR testing should be routinely enquired about at every infant's health care visit. Similarly, any child who presents with malnutrition must be screened for HIV, as reflected in this cohort, where $54.1 \%$ of children were newly diagnosed in hospital despite a background of malnutrition (in 72\%) and previous hospitalisations. However, rapid HIV tests (used for screening or to establish HIV exposure status in infants) have high false-negative rates, especially among young infants. ${ }^{24}$

Despite growing awareness of the benefits of paediatric ART at the time, ART coverage of these hospitalised children was low $(83 \%$ of eligible children were not accessing ART). The short treatment duration and older age of children on ART at $\mathrm{CHBH}$ highlights the fact that few children and even fewer infants had the benefit of early ART initiation. To ensure timeous and equitable access for children, ART must be initiated and monitored at entry levels of the health care system (primary health care clinics). Furthermore, hospitalised, symptomatic HIV-infected children should be fast-tracked for inpatient ART initiation. This measure should be strongly considered for every ART-eligible hospitalised child, and especially for infants $\leq 12$ months of age, who are at highest risk of disease progression and death. ${ }^{23}$ In our study setting (a hospital with an established paediatric ART service), only $4.1 \%$ of ART-naïve, ART-eligible children had treatment commenced as an inpatient, despite weekly ward visits by clinicians from the onsite HIV clinic. In addition, none of those commenced on ART as inpatients were infants, despite this being the age category with the highest case fatality rate. We postulate that multiple hurdles to inpatient ART initiation exist, such as parental illness or death, complex social circumstances, advanced HIV disease and clinician inexperience with or reluctance to commence HAART. Despite these obstacles, clinicians need to be more aggressive in identifying and treating ART-eligible infants and children during ward admission.

\section{OUTCOME OF HOSPITALISATION}

Infectious diseases such as diarrhoea, TB and PCP which are preventable by immunisation, prophylaxis or early ART initiation - accounted for all of the deaths. Dual or multiple concurrent infections are well recognised among HIV-infected children with pneumonia. There were no documented cases in this cohort, but this may simply reflect the lack of aggressive screening for multiple respiratory pathogens. CMV was demonstrated on postmortem specimens from several patients. It was difficult to distinguish CMV infection from disease, additional laboratory testing was limited and ganciclovir treatment was not readily accessible at $\mathrm{CHBH}$ at the time of the study. The contribution of CMV disease to the burden of pneumonia and deaths in this cohort is therefore uncertain.

During the study, only $3 \%$ of HIV-infected children were admitted to ICU/high care; however, they demonstrated a $76.9 \%$ survival rate. Data on ICU candidate selection policies, duration of stay, incidence of complications and long-term morbidity and mortality compared with that of HIV-negative children admitted to ICU were not available. With expanding ART access and improved HIV outcomes, institutional policies for the admission of HIV-infected children to paediatric ICU facilities in South Africa should be reviewed.

AT CHBH from 1992 to 1996 the proportion of paediatric in-hospital mortality accounted for by HIV increased from $6.7 \%$ to $46.1 \%$ and in 2007 (this study) to $58 \%$. This figure shows striking concordance with the 2008 South African statistics from the Countdown to 2015 report, which attributed $57 \%$ of under- 5 mortality to HIVIAIDS (current HIV-attributable mortality is $46 \%$ ). ${ }^{25}$ Despite implementation of PMTCT and paediatric ART programmes, HIV prevalence and in-hospital case fatality rates (among HIV-infected children under 5 years of age) have remained static between 1996 and 2007. Over the same period, however, mortality among uninfected children has declined. HIV-infected children at $\mathrm{CHBH}$ are at a 3-fold increased risk of death compared with HIV-uninfected, HIV-exposed and HIV-unknown children. Hospitalised HIV-infected infants under 12 months of age at $\mathrm{CHBH}$ are a particularly vulnerable group with a high case fatality rate $(17.1 \%)$, and should be prioritised for early ART initiation.

ART status at the time of hospitalisation did not significantly impact on inpatient mortality; however, the median duration of ART in the treatment group was only 2 months, reducing the likelihood of treatment survival benefit. Early hospitalisations after initiation of ART are a well-documented phenomenon, ${ }^{26}$ but no immune reconstitution inflammatory syndrome (IRIS) or ART adverse event-related admissions were documented in this cohort. It is possible that these conditions were unrecognised and thus under-reported owing to lack of experience of hospital staff at that time.

This study has several limitations that may impair its generalisability: a retrospective study design; small sample size; a short study period; and lack of an HIVuninfected comparison group. The large percentage of children (15\%) with unknown HIV status also limits the accuracy of the HIV prevalence data. Missed opportunities among HIV-exposed, status unknown 
infants were also not captured. However, the data provide a 'snap-shot' of HIV impact at a large referral hospital and may reflect commonly encountered challenges to paediatric HIV care provision. Experiences after introduction of PMTCT and ART at $\mathrm{CHBH}$ may provide insights for other institutions struggling to implement best practice guidelines for paediatric HIV care.

\section{CONCLUSION}

HIV remains highly prevalent and contributes to significant in-hospital mortality at $\mathrm{CHBH}$. Multiple missed opportunities for PMTCT, HIV diagnosis and ART initiation were identified, demonstrating the need to monitor and assist with HIV guideline implementation at service delivery level. Interventions to optimise paediatric HIV outcomes should target maternal HIV diagnosis, early infant diagnosis, uptake of CTX prophylaxis and prompt initiation of ART, especially among infants. Hospitalised ART-eligible children should be prioritised for inpatient initiation of ART. Ongoing surveillance of HIV prevalence, disease profile and mortality at $\mathrm{CHBH}$ and other hospitals may be used to identify programmatic problems, plan service improvement interventions and measure progress towards the millennium goal of a twothirds reduction in U5MR by $2015 .^{27}$

Declaration of competing interests. The authors declare that they have no competing interests.

Authors' contributions. All authors contributed to study design, data interpretation and critical revision of the manuscript. AD performed the data collection, data analysis (supervised by $A G$ ) and drafted the manuscript.

Acknowledgements. This research was supported by a Medical Faculty Research Endowment Fund grant from the University of the Witwatersrand. Tammy Meyers is a Fogarty Fellow sponsored by grant No. 5U2RTW007370 and 5U2RTW007373.

\section{REFERENCES}

1. UNAIDS. World Health Organisation. Global report: UNAIDS report on the global AIDS epidemic 2010. http://www.unaids.org/documents/20101123_GlobalReport_ em.pdf (accessed 1 December 2010).

2. Rogerson SR, Gladstone M, Callaghan M, et al. HIV infection among paediatric in-patients in Blantyre, Malawi. Trans R Soc Trop Med Hyg 2004:98(9):544-552.

3. Bradshaw D, Chopra M, Kerber K, et al. Every death counts: use of mortality audit data for decision making to save the lives of mothers, babies, and children in South Africa. Lancet 2008;371:1294-1304.

4. Childlnfo. Monitoring the situation of children and women: Trends in underfive mortality rates (1960 - 2008). 2010. http://www.unicef.org/infobycountry/southafrica statistics.html (accessed 1 December 2010).

5. Bourne DE, Thompson M, Brody LL, et al. Emergence of a peak in early infant mortality due to HIVIAIDS in South Africa. AIDS 2009;23(1):101-106.

6. Newell ML, Coovadia H, Cortina-Borja M, Rollins N, Gaillard P, Dabis F. Mortality of infected and uninfected infants born to HIV-infected mothers in Africa: a pooled analysis. Lancet 2004;364:1236-1243.

7. South African National Department of Health. Guidelines For the Management of HIV-Infected Children. Pretoria: Jacana Media, 2005.

8. Meyers T, Moultrie H, Naidoo K, Cotton M, Eley B, Sherman G. Challenges to pediatric HIV care and treatment in South Africa. J Infect Dis 2007;196(suppl 3):S474-481.

9. Kellerman S, Essajee S. HIV testing for children in resource-limited settings: what are we waiting for? PLoS Med 2010;7(7):e1000285

10. Finlayson H, Eley B. Treatment and outcome of hositalised, very young, HIV-infected children. South African Journal of Child Health 2007;1(4):140-144

11. Patrick M, Stephen C. Child PIP: Making mortality meaningful by using a structured mortality review process to improve the quality of care that children receive in the South African health system. South African Journal of Child Health 2008:2(2):38-42.

12. Zwi K, Pettifor J, Soderlund N, Meyers T. HIV infection and in-hospital mortality at an academic hospital in South Africa. Arch Dis Child 2000;83(3):227-230.

13. Meyers TM, Pettifor JM, Gray GE, Crewe-Brown H, Galpin JS. Pediatric admissions with human immunodeficiency virus infection at a regional hospital in Soweto, South Africa. J Trop Pediatr 2000:46(4):224-230.

14. Statistics South Africa.Mid-year population estimates. 2010. http://www.statssa.gov. za/publications/P0302/P03022010.pdf (accessed 6 November 2011).

15. WHO case definitions of HIV for surveillance and revised clinical staging and immunological classification of HIV-related disease in adults and children. 2007. www.who.int/hiv/pub/guidelines/HIVstaging150307.pdf (accessed 6 November 2011)

16. Fatti GL, Zar HJ, Swingler GH. Clinical indicators of Pneumocystis jiroveci pneumonia (PCP) in South African children infected with the human immunodeficiency virus. Int $\mathrm{J}$ Infect Dis 2006;10(4):282-285.

17. Wijesingha S, Graham S. Evidence behind the WHO guidelines: hospital care for children. What are the clinical indicators of PCP? J Trop Pediatr 2007;53(1):4-7.

18. 2006 WHO Child Growth Standards. www.who.int/childgrowth/standards/en/ (accessed 4 February 2011)

19. Antenatal Survey 2009: National Antenatal Sentinel HIV and Syphilis Prevalence Survey in South Africa, 2009. Pretoria: National Department of Health, 2010. http:// www.hst.org.za/healthstats/13/data (accessed 10 May 2011).

20. Moodley D, Esterhuizen TM, Pather T, Chetty V, Ngaleka L. High HIV incidence during pregnancy: compelling reason for repeat HIV testing. AIDS 2009:23(10):1255-1259.

21. Sherman GG, Jones SA, Coovadia AH, Urban MF, Bolton KD. PMTCT from research to reality - results from a routine service. S Afr Med J 2004;94(4):289-292.

22. Coetzee D, Hilderbrand K, Boulle A, Draper B, Abdullah F, Goemaere E. Effectiveness of the first district-wide programme for the prevention of mother-tochild transmission of HIV in South Africa. Bull World Health Organ 2005:83(7):489494.

23. Violari A, Cotton MF, Gibb DM, et al. Early antiretroviral therapy and mortality among HIV-infected infants. N Engl J Med 2008;359(21):2233-2244.

24. Maree L, van Zyl GU, Lotter SL, de Beer C, Esser MM, Preiser W. Young age a predictor of weak reactivity in a rapid antibody test in infants infected with HIV. J Med Virol 2010;82(8):1314-1317.

25. Countdown to 2015 Maternal, Newborn and Child Health - Country Profiles - South Africa, 2008 Report. http://www.countdown2015mnch.org/documents/ countryprofiles/south africa 20080312.pdf (accessed 12 June 2010).

26. Puthanakit T, Aurpibul L, Oberdorfer $P$, et al. Hospitalization and mortality among HIV-infected children after receiving highly active antiretroviral therapy. Clin Infect Dis 2007;44(4):599-604.

27. Chopra M, Daviaud E, Pattinson R, Fonn S, Lawn JE. Saving the lives of South Africa's mothers, babies, and children: can the health system deliver? Lancet 2009;374(9692):835-846.

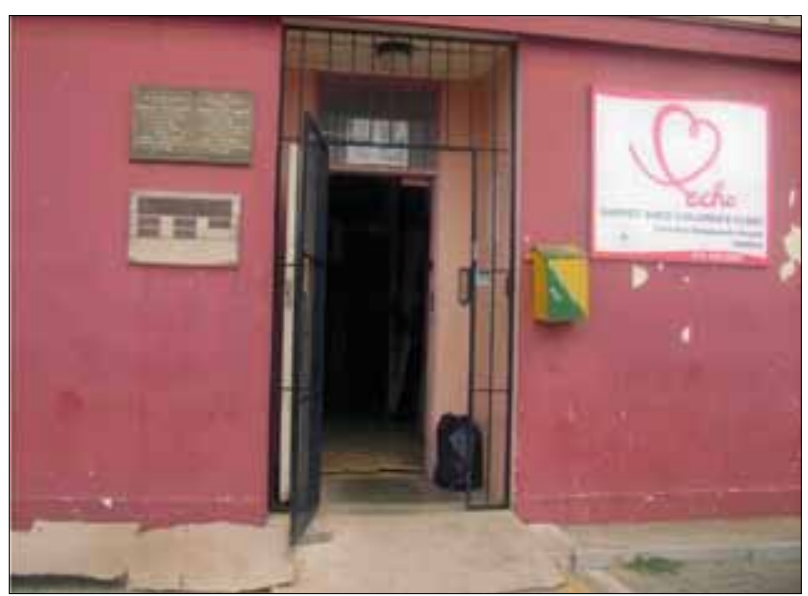

